\title{
Diagnóstico laboratorial da resistência à oxacilina em Staphylococcus aureus
}

\author{
Laboratory diagnosis of oxacillin resistance in Staphylococcus aureus
}

Marcelo J. Mimica'; Caio M. F. Mendes²

unitermos
Staphylococcus aureus
Resistência à oxacilina
Diagnóstico laboratorial

\section{resumo}

Apesar das diversas recomendações disponíveis na literatura sobre o diagnóstico laboratorial da resistência à oxacilina em Staphylococcus aureus, a verdade é que ainda não existe consenso absoluto sobre qual ou quais métodos utilizar e quando utilizar cada um deles. Segundo a literatura, a maioria dos métodos disponíveis apresenta um índice não desprezível de erros diagnósticos. Em casos de isolados de processos infecciosos invasivos, ou de infecções persistentes e/ou refratárias aos antimicrobianos, o uso de um método adicional ou confirmatório pode ser importante. Além disso, a contínua reavaliação desses testes é necessária.

\section{Introdução}

\section{Breve história da evolução da resistência aos antimicrobianos}

Antes da introdução dos antimicrobianos na prática clínica, a letalidade da bacteriemia por Staphylococcus aureus ultrapassava $80 \%$, e mais de $70 \%$ dos pacientes desenvolviam infecções metastáticas ${ }^{(31)}$. No início da década de 1940, com a introdução da penicilina, o prognóstico desses pacientes melhorou bastante ${ }^{(31,32)}$. No entanto, já em 1942 foram relatadas cepas de $S$.

\section{abstract}

Despite guidelines published in the literature, the optimal routine phenotypic method for detecting methicillin tance in Staphylococcus aureus remains controversial. Most available methods have a considerable infections, using an additional or confirmatory method could be important. In addition, the continual evaluation of these tests is necessary.

\section{key words}

Staphylococcus aureus

Oxacillin resistance

Laboratory diagnosis aureus resistentes à penicilina ${ }^{(32)}$. A resistência à penicilina foi reconhecida e aumentou inicialmente em cepas hospitalares e, depois, na comunidade. Contudo, no final dos anos 1960, as taxas de resistência tanto hospitalares como comunitárias chegavam a $90 \%$ e $70 \%$, respectivamente, em algumas regiões da Euro$\mathrm{pa}^{(27)}$. Atualmente, a maioria dos $S$. aureus que causam infecção ou simplesmente colonizam adultos saudáveis é resistente à penicilina ${ }^{(37)}$.

Esse padrão de evolução da resistência, primeiro nos hospitais e posteriormente na comunidade, hoje é um padrão conhecido que recorre a cada introdução e 
uso de antimicrobiano na prática clínica. E a evolução da resistência à oxacilina guarda grande similaridade com esse padrão (Tabela 1) $)^{(4)}$.

Em 1959, o isolamento do ácido 6-aminopenicilânico (6-APA) tornou possível a produção de penicilinas semi-sintéticas. Modificações na cadeia desse precursor da penicilina resultaram em proteção do anel betalactâmico contra a ação hidrolítica das betalactamases. Os primeiros desses agentes antimicrobianos disponíveis para uso clínico foram a oxacilina e a meticilina, que solucionaram temporariamente $o$ problema causado pela resistência do $S$. aureus à penicilina. Porém, o uso desses agentes foi rapidamente seguido pelo surgimento de cepas resistentes em $1961^{(32)}$. Desde então, as taxas de resistência do $S$. aureus à oxacilina aumentaram vertiginosamente $^{(1,4,31,32)}$.

No início, os Staphylococcus aureus meticilino-resistentes (MRSA) estavam restritos a centros médicos de referência e hospitais terciários, mas logo se alastraram para serviços e centros de saúde menores. Estudos de vigilância recentes feitos em várias partes do mundo mostram prevalência variável de MRSA, dependendo do país e, principalmente, do hospital ou setor do hospital estudado. Em alguns locais, já foram relatadas taxas acima de $80 \%(16,37)$.

No entanto, o MRSA não pode mais ser considerado um patógeno relacionado exclusivamente às infecções relacionadas aos serviços de saúde. A partir dos anos 1990 começaram os relatos de infecções por MRSA associadas à comunidade (CA-MRSA: community-associated methicillinresistant Staphylococcus aureus) em pacientes sem fatores de risco identificáveis para aquisição de MRSA, ou seja, não tinham contato freqüente, direto ou indireto, com serviço de saúde que pudesse explicar a infecção por MRSA associada aos cuidados de saúde (health-care associated methicillinresistant Staphylococcus aureus [HCA-MRSA]) $)^{(4,7,21,24)}$. Os CA-MRSA já foram descritos em várias regiões do globo, entre elas o Brasil( ${ }^{(40)}$. Além de infectarem indivíduos sem fatores de risco aparentes, essas cepas têm perfil de resistên- cia e virulência peculiares. Quanto à resistência, são menos resistentes a outras classes de antimicrobianos não-betalactâmicos do que os HCA-MRSA ${ }^{(14,28,36)}$. Em relação à virulência, nessas cepas existe alta prevalência dos genes que codificam a produção da leucocidina de Panton-Valentine (PVL), uma exotoxina associada a infecções de pele e partes moles graves, bem como a pneumonia necrotizante ${ }^{(14,20)}$, embora seu exato papel na patogenia dessas infecções não tenha sido totalmente esclarecido ${ }^{(30,42)}$.

Apesar de diversos estudos publicados sobre possíveis diferenças quanto à virulência entre os $S$. aureus sensíveis à oxacilina e os resistentes, não há, até o momento, evidências conclusivas para essa questão(41).

Para os HCA-MRSA, nas últimas décadas, uma das poucas opções têm sido os glicopeptídeos, principalmente a vancomicina. Mas, em 1996, foi identificado no Japão o primeiro isolado de $S$. aureus com suscetibilidade reduzida à vancomicina (vancomycin-intermediate Staphylococcus aureus [VISA]) ${ }^{(25)}$. Desde então, diversos relatos de isolados de VISA ocorreram no mundo, inclusive no Brasil ${ }^{(33,38)}$.

Em junho de 2002, o primeiro Staphylococcus aureus com resistência plena à vancomicina (vancomycin-resistant S. aureus [VRSA]) foi identificado em Michigan (EUA) ${ }^{(6)}$. Apenas dois meses depois, um segundo VRSA foi isolado na Pensilvânia (EUA) ${ }^{(8)}$ e uma terceira cepa de VRSA foi descrita em Nova lorque (EUA) após mais de um ano e meio ${ }^{(9)}$. $O$ quarto, quinto e sexto isolados de VRSA também foram isolados em Michigan, o último em janeiro de $2006^{(48)}$.

Nesse panorama, o diagnóstico correto da resistência à oxacilina é, mais do que nunca, vital, tanto por razões clínicas quanto epidemiológicas.

\section{Mecanismos de resistência do $S$. aureus à oxacilina}

A resistência à oxacilina no Staphylococcus aureus é determinada, na grande maioria das vezes, pela presença de

\begin{tabular}{lccccc} 
Tabela $\mathbf{1}$ & \multicolumn{5}{c}{ Tempo necessário para taxas de resistência do Staphylococcus aureus à penicilina, vancomicina } \\
e meticilina atingirem & $\mathbf{2 5 \%}$ nos hospitais e na comunidade & \\
\hline Antimicrobiano & Ano de introdução & Anos até primeiro & Anos até taxa de $25 \%$ de & Anos até taxa de $25 \%$ de \\
& na prática clínica & relato de resistência & resistência em hospitais & resistência na comunidade \\
Penicilina & 1941 & $1-2$ & 6 & $15-20$ \\
Vancomicina & 1956 & 40 & $?$ & $?$ \\
Meticilina & 1961 & $<1$ & $25-30$ & $40-50$ (projeção) \\
\hline
\end{tabular}

Adaptado de CHAMBERS, H.F. The changing epidemiology of Staphylococcus aureus? Emerg Infect Dis, 2001. 
um gene localizado no cromossomo, o gene $m e c A^{(5,31)}$. Este gene é responsável pela síntese da penicillin-binding protein (proteína ligadora de penicilina) 2a, ou 2' (PBP2a ou PBP2'), que substitui as outras proteínas ligadoras de penicilina na membrana e têm baixa afinidade não só para a oxacilina como para os outros antimicrobianos betalactâmicos ${ }^{(44)}$. O gene mecA faz parte de uma ilha genômica de resistência chamada staphylococcal cassette chromosome mec (SCCmec), podendo essas ilhas conter também outros genes de resistência a antimicrobianos ${ }^{(31)}$. Enquanto os HCA-MRSA carregam SCCmec dos tipos I a III, os CA-MRSA estão mais associados aos tipos IV e $V^{(13,26)}$. Os tipos IV e V são elementos genéticos menores e com mais mobilidade que os outros. Esses tipos carregam menos genes determinantes de resistência do que os tipos I, II e III. Por isso, os CA-MRSA caracteristicamente tendem a ser menos multirresistentes que os HCA-MRSA, mantendo em geral sensibilidade à clindamicina, por exemplo ${ }^{(14,19,36)}$.

A resistência fenotípica à oxacilina é extremamente variável e depende da expressão do gene mecA. Essa variabilidade é reconhecida como heterorresistência fenotípica, e se caracteriza pelo fato de que de toda população bacteriana heterogeneamente resistente, assim como todas as células, carregam o gene mecA, marcador genotípico da resistência, porém nem todas expressam fenotipicamente sua resistência da mesma forma ${ }^{(32)}$.

Cada cepa de MRSA apresenta um perfil característico da proporção de células que crescem na presença de concentrações específica de oxacilina e de diferentes condições ambientais ${ }^{(31,32)}$. A expressão da resistência à oxacilina no $S$. aureus é regulada por genes homólogos aos reguladores do gene blaZ, determinante da resistência à penicilina. Esses genes (mecl e mecR1) regulam a resposta do mecA aos betalactâmicos de maneira similar à regulação do blaZ pelos genes blaR1 e blal ante a exposição à penicilina ${ }^{(5,31)}$.

Outros mecanismos (mais raros) de resistência à oxacilina que podem ocorrer incluem a superprodução de betalacta- mases e a produção de PBPs habituais (não a PBP2a), porém com graus variados de afinidade pelos betalactâmicos ${ }^{(23,34,51)}$. Esses isolados que elevam muito a produção de betalactamases e/ou com PBPs modificadas, em geral, apresentam resistência fenotípica limítrofe ou de baixo grau ${ }^{(32)}$.

\section{Diagnóstico laboratorial da resistência à oxacilina em $\mathrm{S}$. aureus}

Diversos métodos têm sido utilizados para a detecção da resistência à oxacilina no Staphylococcus aureus. Essa detecção muitas vezes pode ser difícil, principalmente devido ao fenômeno da heterorresistência. Os métodos historicamente mais usados baseiam-se em modificações na tentativa de aumentar a expressão da resistência à oxacilina. Tais modificações incluem:

- incubação a $33-35^{\circ} \mathrm{C}$ ao invés de $37^{\circ} \mathrm{C}$;

- incubação por 24 horas, ao invés de 16-18 horas;

- adição de cloreto de sódio ao meio de cultura(5, 17).

A Tabela 2 mostra de forma resumida os resultados de alguns estudos que avaliaram a acurácia de métodos comumente utilizados para esse diagnóstico. Todos os estudos apresentados utilizaram como gold standard a presença do gene mecA.

Cabe lembrar que os estafilococos diagnosticados como resistentes à oxacilina devem ser relatados como também resistentes a outros betalactâmicos (penicilinas, carbapenêmicos, cefalosporinas e combinações de betalactâmicos com inibidores de betalactamases), independente dos resultados dos testes in vitro com esses antimicrobianos, visto que o mecanismo de resistência é o mesmo e testes in vitro com esses antimicrobianos têm menor acurácia como preditivos da presença do gene mecA do que os testes com oxacilina ou cefoxitina ${ }^{(5,11,53)}$.

\section{Estudos de acurácia (\%) dos métodos fenotípicos para detecção de resistência à oxacilina em}

Tabela 2 Staphylococcus aureus ( $\mathrm{S}=$ sensibilidade; $\mathrm{E}=$ especificidade)

\begin{tabular}{|c|c|c|c|c|c|c|c|c|c|}
\hline \multirow[b]{2}{*}{ Referências } & \multirow[b]{2}{*}{ Isolados } & \multicolumn{2}{|c|}{ Oxacilina } & \multicolumn{2}{|c|}{ Cefoxitina } & \multicolumn{2}{|c|}{ Etest $^{\circledR}$} & \multicolumn{2}{|c|}{ Screening } \\
\hline & & S & E & S & E & S & E & S & E \\
\hline Weller et al. ${ }^{(54)}$ & $\begin{array}{l}21 \mathrm{MRSA} \\
23 \mathrm{MSSA}\end{array}$ & - & - & - & - & 100 & 100 & - & - \\
\hline Cavassini et al..$^{(3)}$ & $\begin{array}{c}80 \text { MRSA } \\
120 \text { MSSA }\end{array}$ & 61,3 & 96,7 & - & - & - & - & 82,5 & 98,3 \\
\hline Kohner et al..(29) & $\begin{array}{l}17 \text { MRSA } \\
24 \text { MSSA }\end{array}$ & 100 & 100 & - & - & - & - & 100 & 100 \\
\hline
\end{tabular}




\begin{tabular}{|c|c|c|c|c|c|c|c|c|c|}
\hline Van Griethuysen et al. ${ }^{(52)}$ & $\begin{array}{l}267 \text { MRSA } \\
296 \text { MSSA }\end{array}$ & - & - & - & - & - & - & 93,6 & 100 \\
\hline Sakoulas et al..$^{(43)}$ & $\begin{array}{l}203 \text { MRSA } \\
107 \text { MSSA }\end{array}$ & - & - & - & - & - & - & 99 & 98,1 \\
\hline Swenson et al. ${ }^{(49)}$ & $\begin{array}{l}19 \text { MRSA } \\
36 \text { MSSA }\end{array}$ & 100 & 89 & - & - & - & - & 90 & 92 \\
\hline Felten et al. ${ }^{(17)}$ & $\begin{array}{l}83 \text { MRSA } \\
69 \text { MSSA }\end{array}$ & 96,4 & 97,1 & 100 & 100 & - & - & 97,6 & 100 \\
\hline Skov et al. ${ }^{(46)}$ & $\begin{array}{l}190 \text { MRSA } \\
267 \text { MSSA }\end{array}$ & 78 & 99 & 100 & 99 & - & - & - & - \\
\hline Fernandes et al. ${ }^{(18)}$ & $\begin{array}{l}180 \text { MRSA } \\
394 \text { MSSA }\end{array}$ & - & - & 100 & 100 & - & - & - & - \\
\hline Pottumarthy et al. ${ }^{(39)}$ & $\begin{array}{l}103 \text { MRSA } \\
100 \text { MSSA }\end{array}$ & 95,6 & 100 & 100 & 100 & - & - & - & - \\
\hline Sharp et al. ${ }^{(45)}$ & $\begin{array}{c}97 \mathrm{MRSA} \\
3 \mathrm{MSSA}\end{array}$ & - & - & 100 & 100 & - & - & 98 & 100 \\
\hline Swenson et al. ${ }^{(50)}$ & $\begin{array}{c}97 \text { MRSA } \\
104 \text { MSSA }\end{array}$ & 98 & 99 & 98 & 100 & - & - & - & - \\
\hline Velasco et al. ${ }^{(53)}$ & $\begin{array}{l}51 \mathrm{MRSA} \\
51 \mathrm{MSSA}\end{array}$ & 94,1 & 100 & 100 & 98 & 94,1 & 100 & 96 & 100 \\
\hline Skov et al. ${ }^{(47)}$ & $\begin{array}{l}50 \text { MRSA } \\
95 \mathrm{MSSA}\end{array}$ & - & - & $98^{*}$ & $100^{*}$ & - & - & - & - \\
\hline Mimica et al. ${ }^{(35)}$ & $\begin{array}{l}50 \text { MRSA } \\
51 \text { MSSA }\end{array}$ & 96 & 98 & $\begin{array}{c}92 \\
98^{*}\end{array}$ & $\begin{array}{c}98 \\
98 *\end{array}$ & 100 & 100 & 100 & 100 \\
\hline Witte et al. ${ }^{(55)}$ & $\begin{array}{c}74 \mathrm{MRSA} \\
117 \mathrm{MSSA} \\
\end{array}$ & 92 & 95 & 96 & 97 & - & - & - & - \\
\hline
\end{tabular}

*Utilizando pontos de corte do Clinical and Laboratory Standards Institute (CLSI) - 2007.

MRSA: Staphylococcus aureus meticilino-resistente; MSSA: Staphylococcus aureus meticilino-sensível.

\section{Testes de disco-difusão}

Para detecção rotineira da resistência à oxacilina os testes de disco-difusão são os mais largamente utilizados. Entre todas as penicilinas penicilinase-estáveis (cloxacilina, dicloxacilina, flucloxacilina, meticilina e nafcilina), a oxacilina é a preferida para testes in vitro utilizando disco-difusão ${ }^{(11)} \mathrm{e}$ vem sendo utilizada por várias décadas, com estudos mostrando acurácias variadas $(3,17,29,35,39,46,49,50)$, provavelmente devido à ocorrência do fenômeno da heterorresistência.

Cavassini et al.(3), estudando cepas heterorresistentes, encontraram sensibilidade do disco de oxacilina de apenas $61,3 \%$, com especificidade de $96,7 \%$. Outro estudo de Skov et al. ${ }^{(46)}$, que avaliou 190 cepas de MRSA e 267 de Staphylococcus aureus meticilino-sensíveis (MSSA), mostrou sensibilidade de $78 \%$, com especificidade de $99 \%$. Porém, Swenson et al. ${ }^{(49)}$, mesmo analisando cepas heterorresistentes, observaram sensibilidade de 100\% (apesar da acurácia de 89\%).
Nos últimos anos, diversos autores têm demonstrado a boa acurácia do teste de disco-difusão com cefoxitina para o diagnóstico da resistência à oxacilina em estafiloco$\cos ^{(17,18,35,39,45,46,50,53)}$. Para os Staphylococcus aureus, os testes com disco de cefoxitina seriam, no mínimo, comparáveis em acurácia aos testes com disco de oxacilina, porém em geral o primeiro teria leitura mais fácil, devido ao maior halo e à possibilidade de ser lido usando luz refletida, e não transmitida, como no caso do disco de oxacilina. Assim, segundo o Clinical and Laboratory Standards Institute (CLSI), que introduziu os pontos de corte de cefoxitina para os estafilococos em 2005, para o S. aureus o disco com cefoxitina deveria ser preferido ao de oxacilina ${ }^{(11)}$. Nesse caso, cabe lembrar que o teste com disco de cefoxitina tem a finalidade de detectar resistência à oxacilina e não a própria cefoxitina.

Apesar das recomendações sobre o uso de diferentes testes de disco-difusão para diagnóstico da resistência à oxacilina 
nos estafilococos, alguns autores $(17,35,46)$ observaram boa acurácia do teste com disco de cefoxitina para o Staphylococcus aureus apenas quando utilizados pontos de corte diferentes dos então (até 2006) padronizados pelo CLSI.

Skov et al. ${ }^{(47)}$ também sugeriram alterações nos pontos de corte para aumentar a sensibilidade desse método. Finalmente, em 2007, o CLSI ${ }^{(11)}$ mudou os pontos de corte para o teste de disco-difusão com cefoxitina, com o objetivo de aumentar a sua acurácia (pontos de corte anteriores: $R$ quando $\leq 19 \mathrm{~mm}$ e $S$ quando $\geq 20 \mathrm{~mm}^{(12)}$; pontos de corte atuais: $R$ quando $\leq 21 \mathrm{~mm}$ e $S$ quando $\left.\geq 22 \mathrm{~mm}^{(11)}\right)$.

Mimica et al. ${ }^{(35)}$, comparando o uso dos pontos de corte anteriores com os atuais do CLSI, observaram que a sensibilidade desse método para detecção da resistência à oxacilina em seus isolados aumentaria de $92 \%$ para $98 \%$, sem prejuízo da especificidade, quando utilizados os novos pontos de corte.

Witte et al. ${ }^{(55)}$ encontraram $96 \%$ de sensibilidade e $97 \%$ de especificidade para o disco de cefoxitina, utilizando uma amostra com isolados mecA-positivos, mas com resistência de baixo grau à oxacilina. Neste estudo, foram utilizados os pontos de corte originais do CLSI. No entanto, mudanças nesses pontos não aumentariam significativamente a sensibilidade desse teste, já que os isolados com resultados falsonegativos para resistência tiveram halos de até $28 \mathrm{~mm}$.

Em 2005, a British Society for Antimicrobial Chemotherapy (BSAC) (2) também incluiu em suas recomendações pontos de corte para a cefoxitina. Esses foram, desde o início, os mesmos valores que o CLSI adota atualmente (novos pontos de corte), porém o disco de cefoxitina recomendado pela BSAC é de $10 \mu \mathrm{g}$, enquanto o recomendado pelo CLSI é de $30 \mu g^{(2,11)}$.

\section{Placa de screening com oxacilina}

Outro teste que pode ser utilizado para detecção da resistência com acurácia geralmente excelente é o teste de screening, com placa contendo ágar Mueller-Hinton suplementado com $4 \%$ de $\mathrm{NaCl}$ e $6 \mu \mathrm{g}$ de oxacilina por

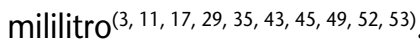

Cavassini et al. ${ }^{(3)}$ encontraram sensibilidade baixa $(82,5 \%)$, porém as 80 cepas de MRSA incluídas no estudo foram previamente diagnosticadas como heterorresistentes. Uma menor sensibilidade da placa de screening (90\%) também foi encontrada por Swenson et al. ${ }^{(49)}$, mas assim como no estudo de Cavassini et al. (3), esses autores analisaram cepas já conhecidas com alto grau de heterorresistência.
As especificidades do método nesses estudos foram de $98,3 \%{ }^{(3)}$ e $92 \%{ }^{(49)}$, respectivamente. Uma grande vantagem do teste é o baixo custo, sobretudo quando se deseja testar vários isolados ao mesmo tempo e apenas para oxacilina, por exemplo, em estudos de vigilância.

\section{Testes para determinação da concentração inibitória mínima}

Há também testes para determinação da concentração inibitória mínima (CIM) da oxacilina para os estafilococos, incluindo métodos de diluição em ágar e em caldo (macrodiluição e microdiluição), e de gradiente de difusão em ágar (Etest ${ }^{\circledR}$ : AB Biodisk, Solna, Suécia). Apesar desses métodos possibilitarem uma avaliação quantitativa da resistência à oxacilina e de serem métodos muitas vezes considerados padrão, os mesmos parecem não ter acurácia significativamente maior (para a presença do gene mecA) do que os testes fenotípicos qualitativos $(22,35,49,53,54)$. No entanto, devido à existência de outros mecanismos de resistência, esses métodos podem e devem ser utilizados como confirmatórios e/ou adjuvantes em isolados nos quais o padrão de suscetibilidade é duvidoso ou de difícil diagnóstico.

\section{Métodos automatizados}

Em relação aos métodos automatizados, como o Vitek e o MicroScan, sua acurácia foi avaliada em alguns estudos. É difícil comparar os resultados desses estudos, visto que há freqüentes mudanças de software e diferentes cartões ou painéis são disponibilizados. Alguns estudos demonstraram acurácia comparável à dos testes de disco-difusão, porém outros evidenciaram sensibilidade e especificidade baixas, se cepas heterorresistentes ou borderline eram $\operatorname{testadas}^{(17,43,49)}$.

\section{Meios cromogênicos}

Outros métodos que podem ser utilizados incluem alguns meios cromogênicos, como MRSASelect (Bio-Rad), MRSA ID (BioMérieux), ORSAB (Oxoid), CHROMagar MRSA, entre outros. Em termos de acurácia esses meios parecem também não oferecer grande vantagem em relação aos demais métodos fenotípicos já discutidos, apesar de serem práticos por possibilitar o isolamento do S. aureus e a caracterização da resistência à oxacilina em apenas um passo $^{(10,15)}$. 


\section{Detecção do gene mecA/PBP2a}

A detecção do gene mecA por métodos moleculares é considerada o método gold standard para avaliação qualitativa da resistência à oxacilina ${ }^{(5)}$. No entanto, esse método não está amplamente disponível nos laboratórios. Um método com acurácia próxima a 100\% é a detecção do produto do gene mecA, a PBP2a, por meio de métodos de aglutinação em látex ${ }^{(3,17,52)}$. Contudo, esses testes ainda são pouco difundidos e utilizados, principalmente devido ao seu custo ${ }^{(18,53)}$.

É importante lembrar que isolados mecA-positivos (ou PBP2a-positivos) devem ser relatados como resistentes à oxacilina. Porém, isolados mecA-negativos (ou PBP2a-negativos) devem ser relatados como sensíveis apenas se a CIM para oxacilina for $\leq 2 \mu \mathrm{g} / \mathrm{ml}$ devido à ocorrência, ainda que rara, de outros mecanismos de resistência (como superprodução de betalactamases e presença de PBPs modificadas), além do mediado pelo mecA. Assim, isolados mecA-negativos (ou PBP2a-negativos), mas com CIM $\geq 4 \mu \mathrm{g} / \mathrm{ml}$, devem ser relatados como resistentes ${ }^{(5,11)}$. Como já comentado, as cepas com PBPs modificadas e as cepas hiperprodutoras de betalactamases costumam, em geral, ter resistência de menor grau ou até limítrofe, quando em comparação com a resistência das cepas mecA-positivas. Essas últimas, apesar de apresentarem com freqüência fenótipo heterogêneo, tendem a ter pelo menos uma subpopulação com resistência de alto $\mathrm{grau}^{(32)}$.

\section{Discussão e conclusões}

As infecções por MRSA se tornaram nos últimos anos um problema cada vez mais complicado, com as novas e preocupantes questões dos Staphylococcus aureus resistentes à oxacilina associados à comunidade ${ }^{(14,28)}$, e dos recentes relatos de resistência intermediária ${ }^{(25)}$ e até mesmo plena à vancomicina ${ }^{(6)}$. Nessa situação, o uso correto de antimicrobianos pode garantir, além de sucesso terapêutico, menor pressão seletiva sobre essas bactérias. Por sua vez, para possibilitar o uso correto de antimicrobianos e de medidas apropriadas de prevenção e controle da infecção, é condição essencial a realização de um diagnóstico de suscetibilidade confiável em laboratório.

Apesar das diversas recomendações disponíveis na literatura sobre o diagnóstico laboratorial da resistência à oxacilina em Staphylococcus aureus, a verdade é que ainda não existe consenso absoluto sobre qual ou quais métodos utilizar e quando utilizar cada um deles. O fenômeno da heterorresistência e a existência de outros mecanismos de resistência complicam ainda mais esse diagnóstico ${ }^{(5,23,31,32,34,51)}$.

Os testes de disco-difusão, incluindo o disco de cefoxitina, utilizando-se os novos pontos de corte do CLSI, e o teste com placa de screening com oxacilina parecem boas opções, tanto em relação aos custos como em relação à acurácia, quando se analisam os dados da literatura. No entanto, esses dados também mostram um índice não desprezível de erros diagnósticos, mesmo com esses métodos. Por isso, em casos de isolados de processos infecciosos invasivos ou de infecções persistentes/refratárias aos antimicrobianos, o uso de um método confirmatório e/ou adicional pode ser importante. Além disso, a contínua reavaliação desses testes por laboratórios em todo o mundo, com as mais variadas condições e características, pode ajudar a garantir um diagnóstico laboratorial cada vez mais confiável.

\section{Referências}

1. BELKUM, A.; VERBRUGH, H. 40 years of methicillin resistant Staphylococcus aureus. MRSA is here to stay-but it can be controlled [editorial]. $\mathrm{Br}$ Med J, v. 323, p. 644-5, 2001.

2. BRITISH SOCIETY FOR ANTIMICROBIAL CHEMOTHERAPY. BSAC Methods for Antimicrobial Susceptibility Testing, version 6.1, February 2007. Disponível em: $<$ http://www.bsac.org.uk/_db/_documents/version_ 6.1.pdf>.

3. CAVASSINI, M. et al. Evaluation of MRSA-Screen, a simple anti-PBP 2a slide latex agglutination kit, for rapid detection of methicillin resistance in Staphylococcus aureus. J Clin Microbiol, v. 37, p. 1591-4, 1999.
4. CHAMBERS, H.F. The changing epidemiology of Staphylococcus aureus? Emerg Infect Dis, v. 7, p. 178-82, 2001.

5. CHAMBERS, H.F. Methicillin resistance in Staphylococci: molecular and biochemical basis and clinical implications. Clin Microbiol Rev, v. 10, p. 781-91, 1997.

6. CHANG, S. et al. Infection with vancomycin-resistant Staphylococcus aureus containing the vanA resistance gene. N Engl J Med, v. 348, p. 1342-7, 2003.

7. CENTERS FOR DISEASE CONTROL AND PREVENTION (CDC). Four pediatric deaths from community-acquired methicillin-resistant Staphylococcus aureus - Minessota 
and North Dakota, 1997-1999. Morb Mortal Wkly Rep, v. 48, p. 707-10, 1999.

8. CENTERS FOR DISEASE CONTROL AND PREVENTION (CDC). Vancomycin-resistant Staphylococcus aureus - Pennsylvania, 2002. Morb Mortal Wkly Rep, v. 51, p. 902, 2002.

9. CENTERS FOR DISEASE CONTROL AND PREVENTION (CDC). Vancomycin-resistant Staphylococcus aureus - New York, 2004. Morb Mortal Wkly Rep, v. 53, p. 322-3, 2004

10. CHERKAOUI, A. et al. Comparison of four chromogenic media for culture based screening of meticillin-resistant Staphylococcus aureus. J Med Microbiol, v. 56, p. 500-3, 2007.

11. CLINICAL AND LABORATORY STANDARDS INSTITUTE (CLSI). Performance standards for antimicrobial susceptibility testing, seventeenth informational supplement, document M100-S17. Wayne, PA, USA: CLSI, 2007.

12. CLINICAL AND LABORATORY STANDARDS INSTITUTE (CLSI). Performance standards for antimicrobial susceptibility testing, sixteenth informational supplement, document M100-S16. Wayne, PA, USA: CLSI, 2006.

13. COOMBS, G.W. et al. Methicillin-resistant Staphylococcus aureus clones, Western Australia. Emerg Infect Dis, v. 12, p. 241-7, 2006.

14. DAUM, R.S. Skin and soft-tissue infections caused by methicillin-resistant Staphylococcus aureus. N Engl J Med, v. 357, p. 380-90, 2007.

15. DIEDEREN, B. et al. Performance of CHROMagar MRSA medium for detection of methicillin-resistant Staphylococcus aureus. J Clin Microbiol, v. 43, p. 1925-7, 2005.

16. FARR, B.M. Prevention and control of methicillin-resistant Staphylococcus aureus infections. Curr Op Infect Dis, v. 17, p. 317-22, 2004.

17. FELTEN, A. etal. Evaluation of three techniques for detection of low-level methicillin-resistant Staphylococcus aureus (MRSA): a disk diffusion method with cefoxitin and moxalactam, the Vitek 2 system and the MRSA-screen latex agglutination test. J Clin Microbiol, v. 40, p. 276671, 2002.

18. FERNANDES, C.J.; FERNANDES, L.A.; COLLIGNON, P. Cefoxitin resistance as a surrogate marker for the detection of methicillin-resistant Staphylococcus aureus. J Antimicrob Chemother, v. 55, p. 506-10, 2005.

19. FRIDKIN, S.K. et al. Methicillin-resistant Staphylococcus aureus in three comunities. N Engl J Med, v. 352, p. 1436-44, 2005.

20. GILLET, Y. et al. Association between Staphylococcus aureus strains carrying gene for Panton-Valentine leukocidin and highly lethal necrotising pneumonia in young immunocompetent patients. Lancet, v. 359, p. 753-9, 2002.

21. GORAK, E.J.; YAMADA, S.M.; BROWN, J.D. Communityacquired methicillin-resistant Staphylococcus aureus in hospitalized adults and children without known risk factors. Clin Infect Dis, v. 29, p. 797-800, 1999.

22. GRADELSKI, E. et al. Correlation between genotype and phenotypic categorization of staphylococci based on methicillin susceptibility and resistance. J Clin Microbiol, v. 39, p. 2961-3, 2001.

23. HACKBARTH, C.J. et al. Point mutation in Staphylococcus aureus PBP2 gene affect penicillin-binding kinetics and are associated with resistance. Antimicrob Agents Chemother, v. 39, p. 103-6, 1995.

24. HEROLD, B.C. et al. Community-acquired methicillinresistant Staphylococcus aureus in children with no identified predisposing risk. JAMA, v. 279, p. 593-8, 1998.

25. HIRAMATSU, K. et al. Methicillin-resistant Staphylococcus aureus clinical strain with reduced vancomycin susceptibility. J Antimicrob Chemother, v. 40, p. 1356, 1997.

26. ITO, T. et al. Novel type $V$ staphylococcal cassette chromosome mec driven by a novel cassette chromosome recombinase, ccrC. Antimicrob Agents Chemother, v. 48, p. 2637-51, 2004.

27. JESSEN, O. etal. Changing staphylococci and staphylococcal infections: a ten-year study of bacteria and cases of bacteremia. N Engl J Med, v. 281, p. 627-35, 1969.

28. KAPLAN, S.L. et al. Three-year surveillance of communityacquired Staphylococcus aureus infections in children. Clin Infect Dis, v. 40, p. 1785-91, 2005.

29. KOHNER, P. et al. Comparison of susceptibility testing methods with mecA gene analysis for determining oxacillin (methicillin) resistance in clinical isolates of Staphylococcus aureus and coagulase-negative Staphylococcus spp. J Clin Microbiol, v. 37, p. 295261, 1999.

30. LABANDEIRA-REY, M. et al. Staphylococcus aureus Panton-Valentine leukocidin causes necrotizing pneumonia. Science, v. 315, p. 1130-3, 2007.

31. LOWY, F.D. Antimicrobial resistance: the example of Staphylococcus aureus. J Clin Invest, v. 111, p. 126573, 2003.

32. MARANAN, M.C. et al. Antimicrobial resistance in Staphylococci: epidemiology, molecular mechanisms and clinical relevance. Infect Dis Clin N Am, v. 11, p. 813-49, 1997.

33. MARLOWE, E.M. et al. Practical strategies for detecting and confirming vancomycin-intermediate Staphylococcus aureus: a tertiary-care hospital laboratory's experience. J Clin Microbiol, v. 39, p. 2637-9, 2001.

34. MCDOUGAL, L.K.; THORNSBERRY, C. The role of betalactamase in staphylococcal resistance to penicillinaseresistant penicillins and cephalosporins. J Clin Microbiol, v. 23, p. 832-9, 1986.

35. MIMICA, M.J. et al. Detection of methicillin resistance in Staphylococcus aureus isolated from pediatric patients: is the cefoxitin disk diffusion test accurate enough? Braz J Infect Dis, v. 11, p. 415-7, 2007.

36. NAIMI, T.S. et al. Comparison of community and health care-associated methicillin-resistant Staphylococcus aureus infection. JAMA, v. 290, p. 2976-84, 2003.

37. OLIVEIRA, D.C.; TOMASZ, A.; DE LENCASTRE, H. Secrets of success of a human pathogen: molecular evolution of pandemic clones of methicillin-resistance Staphylococcus aureus. Lancet Infect Dis, v. 2, p. 180-9, 2002

38. OLIVEIRA, G.A. et al. Isolation in Brazil of nosocomial 
Staphylococcus aureus with reduced susceptibility to vancomycin. Infect Control Hosp Epidemiol, v. 22, p. 443-8, 2001.

39. POTTUMARTHY, S.; FRITSCHE, T.R.; JONES, R.N. Evaluation of alternative disk diffusion methods for detecting mecA-mediated oxacillin resistance in an international collection of staphylococci: validation report from the SENTRY antimicrobial surveillance program. Diagn Microbiol Infect Dis, v. 51, p. 57-62, 2005.

40. RIBEIRO, A. et al. First report of infection with communityacquired methicillin-resistant Staphylococcus aureus in South America. J Clin Microbiol, v. 43, p. 1985-8, 2005.

41. ROZGONYI, F., et al. Is MRSA more virulent than MSSA? Clin Microbiol Infect, v. 13, p. 843-5, 2007.

42. SAID-SALIM, B. etal. Differential distribution and expression of Panton-Valentine leucocidin among communityacquired methicillin-resistant Staphylococcus aureus strains. J Clin Microbiol, v. 43, p. 3373-9, 2005.

43. SAKOULAS, G. et al. Methicillin-resistant Staphylococcus aureus: comparison of susceptibility testing methods and analysis of mecA-positive susceptible strains. J Clin Microbiol, v. 39, p. 3946-51, 2001.

44. SCHITO, G.C. The importance of the development of antibiotic resistance in Staphylococcus aureus. Clin Microbiol Infect, v. 12(supl. 1), p. 3-8, 2006.

45. SHARP, S.E.; WARREN, J.A.; THOMSON, R.B. Cefoxitin disk diffusion screen for confirmation of oxacillinresistant Staphylococcus aureus isolates and utility in the clinical laboratory. Diagn Microbiol Infect Dis, v. 51, p. 69-71, 2005.

46. SKOV, R. et al. Evaluation of a cefoxitin $30 \mu \mathrm{g}$ disc on Iso-Sensitest agar for detection of methicillin-resistant Staphylococcus aureus. J Antimicrob Chemother, v. 52, p. 204-7, 2003.

47. SKOV, R. et al. Phenotypic Detection of methicillin resistance in Staphylococcus aureus by Disk Diffusion Testing and Etest on Mueller-Hinton Agar. J Clin Microbiol, v. 44, p. 4395-9, 2006.

48. SOMSEL, P. The bugs fight back: VRSA, CA-MRSA and QRNG. LabLink, Michigan Department of Community Health - Bureau of Laboratories. Disponível em: <http:// www.michigan.gov/documents/Vol11no1_150647_ 7.pdf (2006)>.

49. SWENSON, J.M. et al. Performance of eigth methods, including two new rapid methods, for detection of oxacillin resistance in a challenge set of Staphylococcus aureus organisms. J Clin Microbiol, v. 39, p. 3785-8, 2001.

50. SWENSON, J.M.; TENOVER, F.C.; CEFOXITIN DISK STUDY GROUP. Results of disk diffusion testing with cefoxitin correlate with presence of mecA in Staphylococcus spp. J Clin Microbiol, v. 43, p. 3818-23, 2005.

51. TOMASZ, A. et al. New mechanism for methicillin resistance in Staphylococcus aureus: clinical isolates that lack the PBP 2a gene and contain normal penicillinbinding proteins with modified penicillin-binding capacity. Antimicrob Agents Chemother, v. 33, p. 1869-74, 1989.

52. VAN GRIETHUYSEN, A. et al. Rapid slide latex agglutination test for detection of methicillin resistance in Staphylococcus aureus. J Clin Microbiol, v. 37, p. 2789-92, 1999.

53. VELASCO, D. etal. Evaluation of different methods for detecting methicillin (oxacillin) resistance in Staphylococcus aureus. J Antimicrob Chemother, v. 55, p. 379-82, 2005.

54. WELLER, T.M.A. et al. Methicillin susceptibility testing of staphylococci by Etest and comparison with agar dilution and mecA detection. J Antimicrob Chemother, v. 39, p. 251-3, 1997.

55. WITTE, W.; PASEMANN, B.; CUNY, C. Detection of low-level oxacillin resistance in mecA-positive Staphylococcus aureus. Clin Microbiol Infect, v. 13, p. 408-12, 2007. 